\title{
Recent Neutrino Cross Section Measurements from MicroBooNE
}

\author{
Steven Gardiner*广 \\ Fermi National Accelerator Laboratory \\ Batavia, IL 60510, USA \\ E-mail: gardiner@fnal.gov
}

\begin{abstract}
Next-generation precision measurements of neutrino properties will require carefully controlled systematic uncertainties, including theoretical uncertainties in models of neutrino-nucleus interactions. Despite their strengths as a neutrino detector technology, liquid argon time projection chambers (LArTPCs) employ a target material (argon) for which neutrino scattering cross sections remain largely unmeasured. To better constrain neutrino interaction uncertainties for future argon-based neutrino oscillation experiments, the MicroBooNE LArTPC experiment is pursuing a wide-ranging program of cross section measurements. These proceedings present some of the first physics results from that effort, including studies of inclusive charged-current $v_{\mu}$ interactions, neutral pion production, and multi-proton final states.
\end{abstract}

XXIX International Symposium on Lepton Photon Interactions at High Energies - LeptonPhoton2019 August 5-10, 2019

Toronto, Canada

\footnotetext{
* Speaker.

${ }^{\dagger}$ For the MicroBooNE collaboration
} 


\section{Introduction}

Neutrino oscillations provide a means of experimentally studying some of the highest-priority questions on the frontier of contemporary particle physics, including (1) the degree to which $\mathrm{CP}$ violation exists in the lepton sector (with implications for theories of leptogenesis), (2) whether $v_{3}$ is the heaviest or the lightest of the three known neutrino mass eigenstates, and (3) whether the existence of one or more species of sterile neutrinos, which do not participate in weak interactions, is needed to explain several anomalies [1] reported by short-baseline neutrino experiments. Definitively answering these questions will require an unprecedented level of precision, including percent-level control of systematic uncertainties [2]. A key challenge for neutrino oscillation measurements is that, because neutrinos cannot be observed directly, their energies must be inferred from detected final-state particles on an event-by-event basis. Translation of observed event topologies to neutrino energies is routinely done for oscillation experiments using detector response simulations in tandem with an event generator, a computer code implementing a probabilistic model of neutrino interaction physics. For experiments employing complex nuclear targets at accelerator energies (hundreds of $\mathrm{MeV}$ to tens of $\mathrm{GeV}$ ), theoretical uncertainties on neutrino-nucleus cross section models are typically a leading source of systematic error. Neutrino cross section data thus serve as a necessary constraint on the theoretical models (and their implementation in event generators) needed to deliver oscillation-based measurements of neutrino properties.

Due to their capabilities for calorimetry, high-resolution tracking, low detection thresholds, and particle discrimination, ${ }^{1}$ liquid argon time projection chambers (LArTPCs) represent an attractive detector technology for use in neutrino oscillation experiments. In the coming years, two large experimental efforts hosted by Fermilab, DUNE [4] and the Short Baseline Neutrino program [5], will employ LArTPCs in pursuit of precision oscillation measurements. Despite growing interest in liquid argon as a neutrino detection medium, few measurements of neutrino-argon cross sections have been made to date. While experimental data for other target nuclei, including relatively wellstudied carbon, can provide useful information for LArTPC experiments, theoretically-challenging nuclear physics effects complicate the interpretation of such results for the argon case. Direct measurements of neutrino-argon interactions, such as those described in these proceedings, are therefore highly desirable in order for future argon-based neutrino oscillation experiments to achieve the precision needed for their ambitious physics goals.

\section{MicroBooNE}

The MicroBooNE detector [6], which operates in the Booster Neutrino Beam at Fermilab, is currently the longest-running LArTPC experiment in a neutrino beam, having started operations in October 2015. The 85-ton single-phase active volume of MicroBooNE is rectangular and measures $10.36 \mathrm{~m}$ long in the beam direction, $2.32 \mathrm{~m}$ tall, and $2.56 \mathrm{~m}$ wide. Ionization electrons drift along the width of the detector in a $273 \mathrm{~V} / \mathrm{cm}$ electric field toward three wire planes with $3 \mathrm{~mm}$ pitch, reaching the anode within $2.3 \mathrm{~ms}$. MicroBooNE is also instrumented with thirty-two 8-inch photomultiplier tubes (PMTs) which image prompt scintillation light. The PMT data are used to trigger the detector and to enhance rejection of cosmic-ray backgrounds.

\footnotetext{
${ }^{1}$ Particularly between electrons and photons [3], the latter of which may mimic a $v_{e}$ appearance signal.
} 
The primary physics goal of the MicroBooNE experiment is to investigate the origin and nature of an excess of low-energy electron-like events observed in short-baseline oscillation measurements by the MiniBooNE experiment [7]. In addition to this flagship analysis targeting the MiniBooNE anomaly, MicroBooNE is also pursuing high-statistics measurements of neutrino-argon cross sections. The remainder of these proceedings presents two cross section measurements recently completed by the collaboration and concludes with a discussion of a third ongoing analysis.

\section{Inclusive $v_{\mu}$ charged-current cross section}

An inclusive charged-current (CC) neutrino scattering measurement, in which one considers only the outgoing charged lepton irrespective of any other final-state particles, is among the most straightforward to execute and understand from an experimental point of view. Comparisons of such data between experiments are relatively uncomplicated due to the clear signal definition, and the event selection developed for such an analysis can serve as a foundation for subsequent investigations of more complicated event topologies.

Despite their apparent simplicity, however, $\mathrm{CC}$ inclusive cross sections represent a mixture of several underlying reaction modes. In the case of MicroBooNE, which operates in a neutrino beam (the BNB) with a mean energy of $0.8 \mathrm{GeV}$, quasi-elastic (QE) scattering is dominant, but there are significant contributions from resonance production (RES), deep inelastic scattering (DIS), and interactions involving two-nucleon meson-exchange currents (MEC). Neutrino event generators strive to accurately simulate all of these scattering processes, and MicroBooNE's recent measurement of the CC inclusive cross section on argon for muon neutrinos [8] thus provides a wideranging test of this modeling effort.

Because MicroBooNE is situated only slightly below ground level with no soil overburden, it is exposed to a large flux of cosmic-ray muons, with roughly twenty expected to arrive within each $4.8 \mu$ S TPC readout window. Distinguishing between these and the neutrino-induced muons that comprise the $\mathrm{CC}$ inclusive signal is a key analysis challenge. Using a variety of selection criteria, including matching optical flashes detected by the PMTs to reconstructed particle tracks in the TPC, the cosmic background is reduced by more than $99.9 \%$. A final efficiency of $57.2 \%$ and purity of $50.4 \%$ are achieved for the event selection, with full angular acceptance. To enable the inclusion of muons in the analysis that are not fully contained in the detector, a novel technique based on multiple Coulomb scattering (MCS) is used to measure the muon momentum [9].

Figure 1 shows a selected subset of MicroBooNE's CC inclusive results, together with a comparison to four theory predictions calculated using recent versions of the GENIE [10], GiBUU [11], and NuWro [12] event generators. This first double-differential measurement of neutrino scattering on argon is reported as a function of reconstructed muon momentum and scattering angle. To aid future comparisons to theoretical calculations of this cross section, a "forward-folding" matrix that transforms from true to reconstructed kinematic variables is published together with the cross section data. Although significant disagreements are observed between the predictions and the data for all four generator configurations tested (with the lowest $\chi^{2}$ value of 103.9 for 42 bins achieved by the GENIE v3.0.6 model), the high-momentum bins for $0.86 \leq \cos \theta_{\mu}<0.94$ and $0.94 \leq \cos \theta_{\mu} \leq 1$ strongly disfavor the GENIE v2 model with empirical MEC. The reduced tension seen in this region of phase space for the other models is driven by their adoption of a local 
Fermi gas description of the initial nuclear state (as opposed to a global Fermi gas in GENIE v2) and, to a lesser extent, corrections for long-range nucleon correlations included in the GENIE v3 and NuWro models via the random phase approximation (RPA).
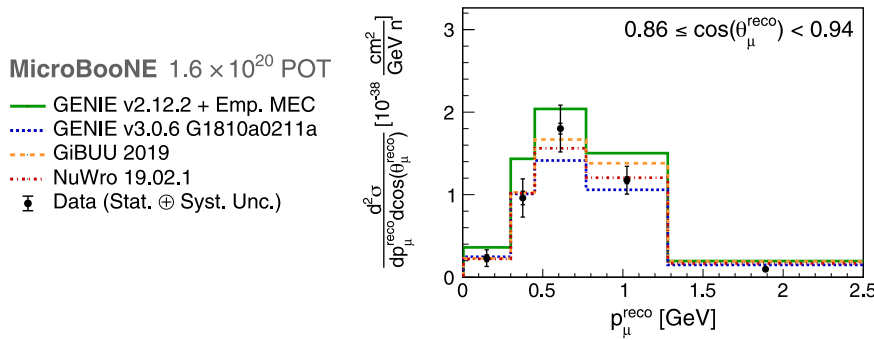

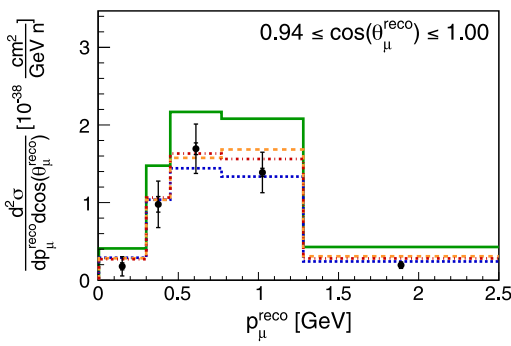

Figure 1: Double-differential $v_{\mu} \mathrm{CC}$ inclusive cross section on argon evaluated per nucleon as a function of the reconstructed muon momentum $p_{\mu}^{\text {reco }}$ and scattering angle $\cos \theta_{\mu}^{\text {reco }}$. The data are compared to theoretical calculations produced using three neutrino event generators. Only the most forward-going angular bins are shown here, with the full results available in ref. [8].

\section{Charged-current neutral pion production cross section}

In addition to playing a critical role in the estimation of reacting neutrino energies in oscillation experiments, event generators are also routinely employed to estimate beam-correlated backgrounds. For oscillation measurements that rely on $v_{e}$ appearance in a predominantly $v_{\mu}$ beam, a problematic source of background is the production of neutral pions $\left(\pi^{0}\right)$ by neutral-current (NC) neutrino-nucleus interactions. High-energy photons produced by decays of these pions can be difficult to distinguish from the electrons that represent the $v_{e}$ appearance signal. Although CC $\pi^{0}$ production differs from $\mathrm{NC}$ in the theoretical description of the primary neutrino interaction, it shares many nuclear physics effects in common with the NC case. MicroBooNE's recent measurement of $v_{\mu} \mathrm{CC} \pi^{0}$ production on argon [13], the heaviest target nucleus for which this process has been studied to date, provides a helpful test of models of these nuclear effects.

To identify CC events involving a $\pi^{0}$ in the final state, this analysis makes use of the CC inclusive event selection described previously as a pre-filter. Passing events are subjected to a second round of reconstruction designed to identify electromagnetic (EM) particle showers associated with the neutrino interaction vertex. The fully-automated EM shower reconstruction chain, the first of its kind for a LArTPC, is described in detail in ref. [14]. To reduce statistical errors in the final cross section result, the $v_{\mu} \mathrm{CC} \pi^{0}$ selection requires at least one photon candidate (out of two expected for the decay $\pi^{0} \rightarrow \gamma \gamma$ ) to be successfully reconstructed. As a cross-check of this procedure, a subsample of events containing two photon candidates is also studied and found to give identical results within statistical uncertainties.

The right panel of fig. 2 shows MicroBooNE's measurement of the flux-integrated $v_{\mu}$ CC $\pi^{0}$ production cross section on argon, $\langle\sigma\rangle_{\Phi}=1.9 \pm 0.2$ (stat) \pm 0.6 (syst) $\times 10^{-38} \mathrm{~cm}^{2} / \mathrm{Ar}$, compared to four theoretical predictions from the GENIE and NuWro event generators. Similar plots are given in the neighboring panels for previous measurements on lighter nuclear targets: deuterium from the ANL bubble chamber [15] and hydrocarbon from MiniBooNE [16]. The solid red lines in the figure show predictions from NuWro. The solid blue and pink lines correspond to different 
GENIE configurations which use the Rein-Sehgal [17] (RS) and Berger-Sehgal [18] (BS) models of resonant pion production, respectively. Both of these GENIE predictions share the same description of hadronic final-state interactions (FSIs) in the nuclear medium. The dashed blue line shows the GENIE RS prediction with FSIs disabled, highlighting their increasing importance with rising nuclear target mass. For the three measurements shown here, the NuWro, GENIE RS, and GENIE BS predictions all show close agreement (within 1.2 $\sigma$ ) with the data, confirming good performance of these generators for predicting total $v_{\mu} \mathrm{CC} \pi^{0}$ cross sections for light- and medium-mass target nuclei.
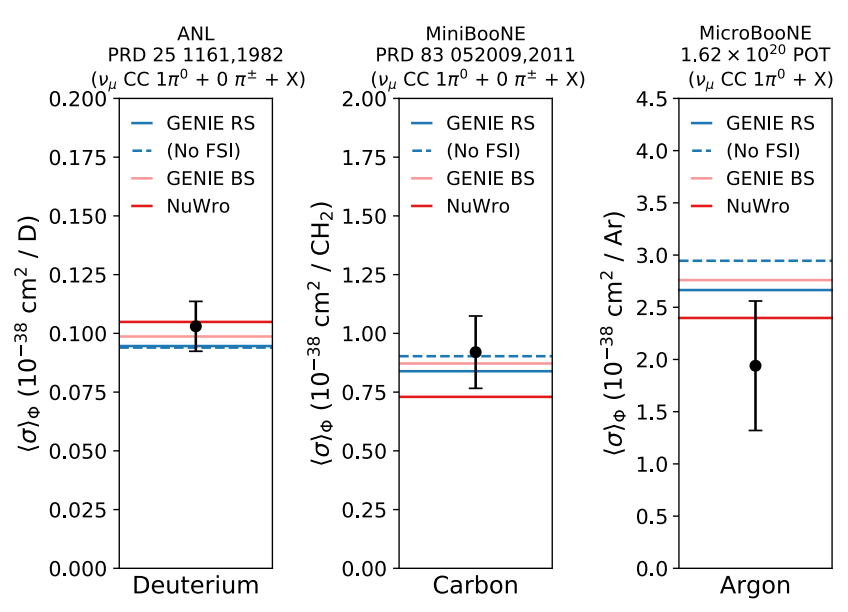

Figure 2: Measured flux-integrated $v_{\mu} \mathrm{CC} \pi^{0}$ total cross section for ANL, MiniBooNE, and MicroBooNE. The data are compared to theoretical predictions calculated using NuWro (solid red) and three GENIE configurations: the default RS model (solid blue), the RS model with FSIs disabled (dashed blue), and the alternative BS model model (solid pink). Figure from ref. [13].

\section{Studies of proton production}

In recent years, increasing attention has been paid to the role of multinucleon processes in neutrino scattering cross sections. Due to their capacity for detailed event reconstruction, LArTPCs offer a promising means of constraining models of these processes via studies of hadronic final states. Having demonstrated the ability to reconstruct neutrino scattering events involving multiple charged particle tracks [19], including multiple protons (see fig. 3), the MicroBooNE collaboration is actively pursuing cross section analyses studying CC $N$-proton (for $N \geq 1$ ) and specifically CC two-proton (CC2p) event topologies [20].

Neutrino event generator predictions for the multiplicity and kinematic distributions of finalstate protons can differ substantially. A clear example of this can be seen in the three panels of fig. 4, which compare theoretical calculations performed using NuWro and two configurations of GENIE v2. For each model set, the distribution of the cosine of the opening angle $\theta_{p 1 p 2}$ between the final-state protons in simulated CC2p events is shown. Figure 5 shows a preliminary MicroBooNE measurement of this distribution, which somewhat favors the alternative GENIE model over the default one. However, it should be noted that the presented number of events is low compared to the available data. The anticipated final results of the CC2p analysis, which will include a 


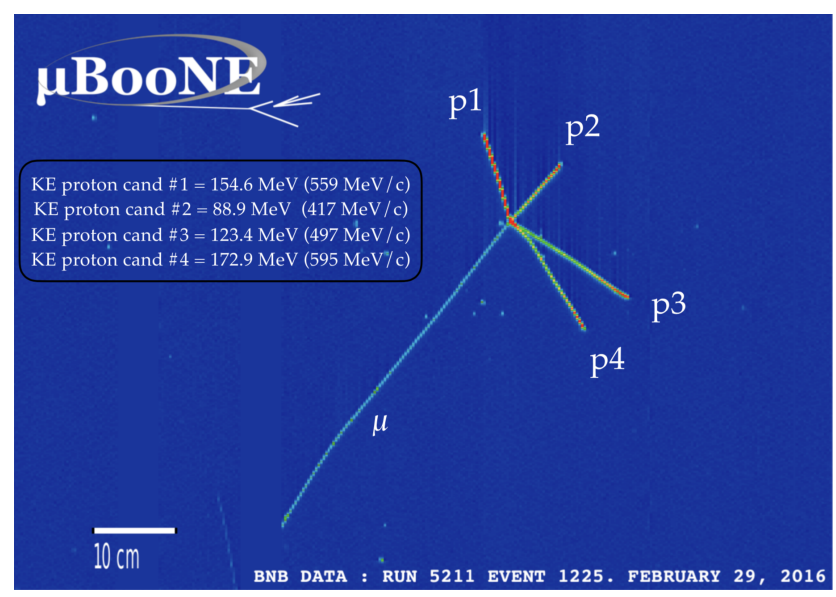

Figure 3: Event display showing an on-beam data event in MicroBooNE. The muon candidate (which is the longest track shown) is accompanied by four proton candidates, each of which is labeled with its reconstructed kinetic energy and momentum. Figure from ref. [20].

full assessment of systematic uncertainties (in contrast to the purely statistical uncertainties shown here), will contribute to efforts to discriminate between the competing nuclear physics models that shape the differing generator predictions.
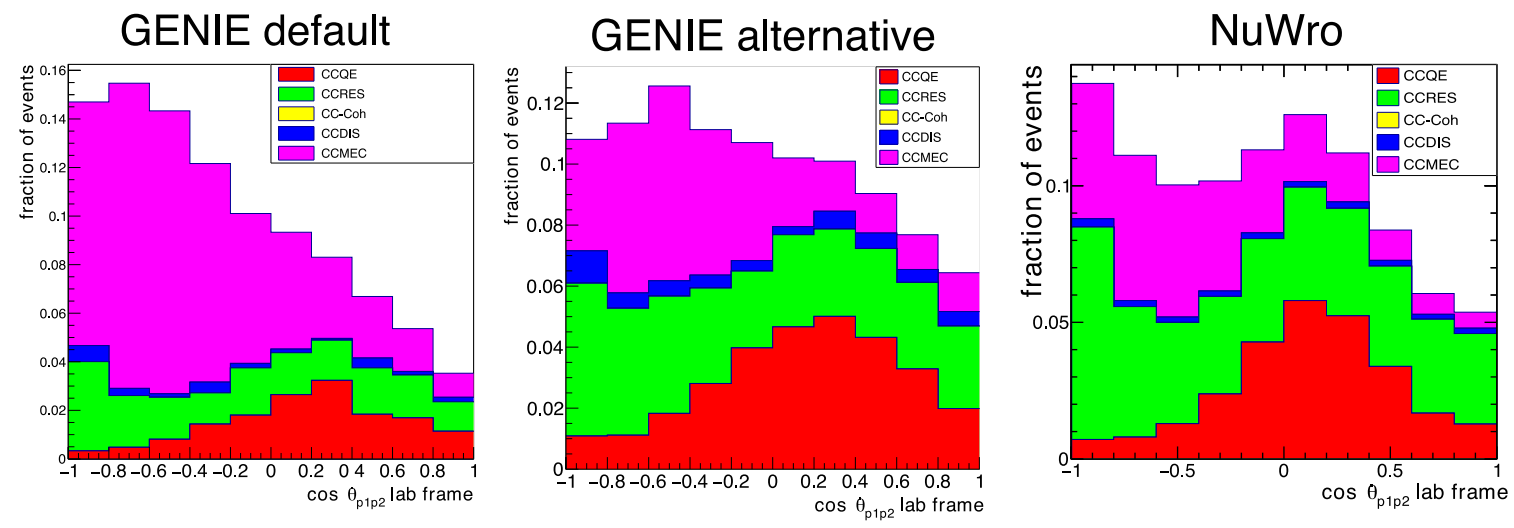

Figure 4: LEFT: GENIE v2 prediction (using the default model set) for the distribution of the cosine of the opening angle $\theta_{p 1 p 2}$ between the two final-state protons in true CC2p events in MicroBooNE. The stacked histograms represent distinct reaction modes. CENTER: The CC2p opening angle distribution predicted by GENIE v2 using an alternative model set. RIGHT: The CC2p opening angle distribution predicted by NuWro. Figures from ref. [20].

\section{Conclusion}

Alongside its primary mission to investigate the low-energy excess seen by MiniBooNE, the MicroBooNE experiment is providing some of the first detailed measurements of neutrino-argon scattering. The early results communicated in these proceedings, as well as a broad program of anticipated future measurements, will serve as a valuable resource for neutrino oscillation experiments in the years to come. 


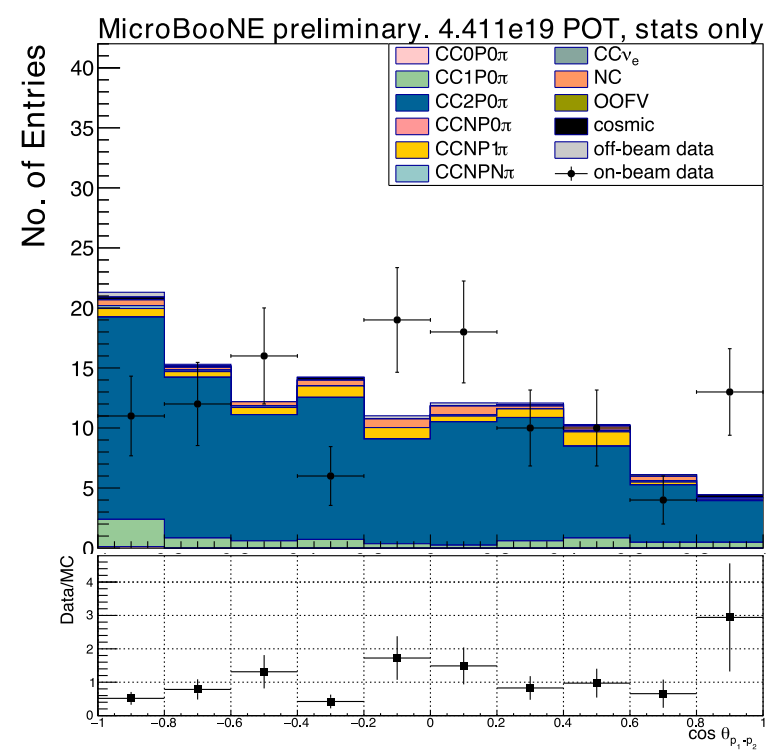

Figure 5: Preliminary measurement of the opening angle distribution for CC two-proton events in MicroBooNE. The data points, rendered in black with purely statistical error bars, are shown together with simulation results produced using the GENIE v2 default model set. Figure from ref. [20].

\section{References}

[1] C. Giunti and T. Lasserre, eV-Scale Sterile Neutrinos, Annual Review of Nuclear and Particle Science 69 (2019) [1901.08330].

[2] NUSTEC collaboration, NuSTEC white paper: Status and challenges of neutrino-nucleus scattering, Progress in Particle and Nuclear Physics 100 (2018) 1 [1706.03621v2].

[3] ARgONEUT collaboration, First observation of low energy electron neutrinos in a liquid argon time projection chamber, Phys. Rev. D 95 (2017) 072005 [1610.04102].

[4] DUNE collaboration, The DUNE Far Detector Interim Design Report Volume 1: Physics, Technology, and Strategies, 1807.10334.

[5] MicroBooNE, LAr1-ND, and ICARUS-WA104 collaborations, A proposal for a three detector short-baseline neutrino oscillation program in the Fermilab Booster Neutrino Beam, 1503.01520.

[6] MicroBooNE collaboration, Design and construction of the MicroBooNE detector, J. Instrum. 12 (2017) P02017 [1612.05824].

[7] MiniBooNE collaboration, Significant excess of electronlike events in the MiniBooNE short-baseline neutrino experiment, Phys. Rev. Lett. 121 (2018) 221801 [1805.12028].

[8] MicroBooNE collaboration, First measurement of inclusive muon neutrino charged current differential cross sections on argon at $E_{v} \sim 0.8 \mathrm{GeV}$ with the MicroBooNE detector, Phys. Rev. Lett. 123 (2019) 131801 [1905.09694].

[9] MicroBooNE collaboration, Determination of muon momentum in the MicroBooNE LArTPC using an improved model of multiple coulomb scattering, J. Instrum. 12 (2017) P10010 [1703.06187].

[10] C. Andreopoulos et al., The GENIE Neutrino Monte Carlo Generator, Nuclear Instruments and Methods A614 (2010) 87 [0905.2517]. 
[11] O. Buss, T. Gaitanos, K. Gallmeister, H. van Hees, M. Kaskulov, O. Lalakulich et al., Transport-theoretical description of nuclear reactions, Physics Reports 512 (2012) 1 [1106.1344].

[12] T. Golan, J. Sobczyk and J. Żmuda, NuWro: the Wrocław Monte Carlo generator of neutrino interactions, Nuclear Physics B - Proceedings Supplements 229-232 (2012) 499.

[13] MicroBooNE collaboration, First measurement of $v_{\mu}$ charged-current $\pi^{0}$ production on argon with the MicroBooNE detector, Phys. Rev. D 99 (2019) 091102 [1811. 02700 ].

[14] MicroBooNE collaboration, Reconstruction and measurement of $O(100)$ MeV energy electromagnetic activity from $\pi^{0} \rightarrow \gamma \gamma$ decays in the MicroBooNE LArTPC, 1910.02166.

[15] G. M. Radecky, V. E. Barnes, D. D. Carmony et al., Study of single-pion production by weak charged currents in low-energy vd interactions, Phys. Rev. D 25 (1982) 1161.

[16] MiniBooNE collaboration, Measurement of neutrino-induced charged-current charged pion production cross sections on mineral oil at $E_{v} \sim 1 \mathrm{GeV}$, Phys. Rev. D 83 (2011) 052007 [1011.3572].

[17] D. Rein and L. M. Sehgal, Neutrino-excitation of baryon resonances and single pion production, Ann. Phys. 133 (1981) 79.

[18] C. Berger and L. M. Sehgal, Partially conserved axial vector current and coherent pion production by low energy neutrinos, Phys. Rev. D 79 (2009) 053003 [0812.2653].

[19] C. Adams, R. An, J. Anthony et al., Comparison of $v_{\mu}-$ Ar multiplicity distributions observed by MicroBooNE to GENIE model predictions, The European Physical Journal C 79 (2019) 248 [1805.06887].

[20] MicroBooNE collaboration, "Selection of $v_{\mu}$ charged-current induced interactions with $N>0$ protons and performance of events with $N=2$ protons in the final state in the MicroBooNE detector from the BNB." MicroBooNE Public Note 1056, October, 2018. 